\title{
Heavy Flavor Contributions to DIS Structure Functions at $O\left(\alpha_{s}^{3}\right)$
}

\author{
Isabella Bierenbaum ${ }^{a, b}$, Johannes Blümlein ${ }^{a *}$ and Sebastian Klein ${ }^{c}$ \\ ${ }^{a}$ DESY, Zeuthen, Platanenallee 6, D-15735 Zeuthen, Germany. \\ ${ }^{b}$ Instituto de Física Corpuscular, CSIC-Universitat de València, \\ Apartado de Correos 22085, E-46071 Valencia, Spain. \\ ${ }^{c}$ Institut für Theoretische Physik E, RWTH Aachen University, D-52056 Aachen Germany.
}

\begin{abstract}
We calculate moments of the $O\left(\alpha_{s}^{3}\right)$ heavy flavor contributions to the Wilson coefficients of the structure function $F_{2}\left(x, Q^{2}\right)$ in the region $Q^{2} \gg m^{2}$. The massive Wilson coefficients are obtained as convolutions of massive operator matrix elements (OMEs) and the known light flavor Wilson coefficients. The calculation of moments of the massive OMEs involves a first independent recalculation of moments of the fermionic contributions to all 3-loop anomalous dimensions of the unpolarized twist-2 local composite operators stemming from the light-cone expansion. The expressions for the massive OMEs are now known for general values of the Mellin variable $N$ apart of one coefficient in the constant term, for which only a series of moments is computed.
\end{abstract}

RADCOR 2009 - 9th International Symposium on Radiative Corrections (Applications of Quantum Field Theory to Phenomenology),

October 25 - 302009

Ascona, Switzerland

${ }^{*}$ Speaker. 


\section{Introduction}

Deep-inelastic scattering (DIS) of charged or neutral leptons off proton and deuteron targets, in the region of large enough values of the gauge boson virtuality $Q^{2}=-q^{2}$, allows to measure the leading twist parton densities of the nucleon, the QCD-scale $\Lambda_{\mathrm{QCD}}$, and the strong coupling constant $a_{s}\left(Q^{2}\right)=\alpha_{s}\left(Q^{2}\right) /(4 \pi)$, to high precision. For unpolarized DIS via single photon exchange, the double-differential cross section can be expressed in terms of two inclusive structure functions $F_{2, L}\left(x, Q^{2}\right)$. These decompose for twist $\tau=2$ into a Mellin convolution of non-perturbative massless parton densities $f_{j}\left(x, \mu^{2}\right)$ and the perturbative Wilson coefficients $\mathscr{C}_{j,(2, L)}\left(x, Q^{2} / \mu^{2}, m_{k}^{2} / \mu^{2}\right)$. The latter describe the hard scattering of the photon with a massless parton. They are given by the sum of the purely light - denoted by $C_{j,(2, L)}$ - and heavy flavor contributions, $\mathrm{H}_{j,(2, L)}$. Here $k=c, b$ and $j=q, g$, depending on the type of process one considers. $x$ denotes the Bjorken scaling variable. Especially in the region of smaller values of Bjorken- $x$, the structure functions contain large $c \bar{c}$-contributions of up to $20-40 \%$, denoted by $F_{2, L}^{c \bar{c}}\left(x, Q^{2}\right)$. The perturbative heavy flavor Wilson coefficients corresponding to these structure functions are known at NLO semi-analytically in $x-$ space [1]. Due to the size of the heavy flavor corrections, it is necessary to extend the description of these contributions to $O\left(a_{s}^{3}\right)$, and thus to the same level which has been reached for the massless Wilson coefficients [2].

A calculation of these quantities in the whole kinematic range at NNLO seems to be out of reach at present. However, in the limit of large virtualities $Q^{2}, Q^{2} \gtrsim 10 m_{c}^{2}$ in the case of $F_{2}^{c \bar{c}}\left(x, Q^{2}\right)$, one observes that $F_{2 . L}^{c \bar{c}}\left(x, Q^{2}\right)$ are very well described by their asymptotic expressions [3] neglecting power corrections in $\mathrm{m}^{2} / Q^{2}$, cf. also [4]. In this kinematic range, one can calculate the heavy flavor Wilson coefficients analytically. This has been done for $F_{2}^{c \bar{c}}\left(x, Q^{2}\right)$ to 2-loop order in [3, 5] and for $F_{L}^{c \bar{c}}\left(x, Q^{2}\right)$ to 3-loop order in [6]. Note that in the latter case, the asymptotic result becomes valid only at much higher values of $Q^{2}$. The asymptotic expressions are obtained by a factorization of the heavy quark Wilson coefficients into a Mellin convolution of massive OMEs $A_{j k}$ and the massless Wilson coefficients $C_{j, i}$, if one heavy quark flavor of mass $m$ and $n_{f}$ light flavors are considered. In the present paper, we report on the calculation of the massive OMEs $A_{j k}$ to 3-loop order for fixed even moments of the Mellin variable $N$, cf. [7] for details. We further calculate the OMEs which are required to define heavy quark parton densities in the variable flavor number scheme [8]. We also obtain moments of the terms $\propto T_{F}$ of the 3-loop unpolarized anomalous dimensions $\gamma_{i j}$. Our results agree with those obtained in [9]. Since the present calculation is completely independent by method, formalism, and codes, it provides a strong check on the previous results.

\section{Heavy Flavor Operator Matrix Elements}

The heavy flavor Wilson coefficients for a single massive quark may be expressed as

$$
\mathrm{H}_{j,(2, L)}^{\mathrm{S}, \mathrm{PS}, \mathrm{NS}}\left(x, \frac{Q^{2}}{\mu^{2}}, \frac{m^{2}}{\mu^{2}}\right)=H_{j,(2, L)}^{\mathrm{S}, \mathrm{PS}}\left(x, \frac{Q^{2}}{\mu^{2}}, \frac{m^{2}}{\mu^{2}}\right)+L_{j,(2, L)}^{\mathrm{S}, \mathrm{PS}, \mathrm{NS}}\left(x, \frac{Q^{2}}{\mu^{2}}, \frac{m^{2}}{\mu^{2}}\right)
$$

where the photon couples to a light $(L)$ or heavy $(H)$ quark line, respectively. Further $S$ stands for the flavor-singlet contributions, which are separated into a pure-singlet (PS) and non-singlet (NS) part via $\mathrm{S}=\mathrm{PS}+\mathrm{NS}$. The factorization formula for the inclusive Wilson coefficients reads in 
Mellin space, [3, 8],

$$
\mathscr{C}_{j,(2, L)}^{\mathrm{S}, \mathrm{PS}, \mathrm{NS}, \mathrm{as}}\left(N, n_{f}, \frac{Q^{2}}{\mu^{2}}, \frac{m^{2}}{\mu^{2}}\right)=\sum_{i} A_{i j}^{\mathrm{S}, \mathrm{PS}, \mathrm{NS}}\left(N, n_{f}, \frac{m^{2}}{\mu^{2}}\right) C_{i,(2, L)}^{\mathrm{S}, \mathrm{PS} \mathrm{NS}}\left(N, n_{f}+1, \frac{Q^{2}}{\mu^{2}}\right) .
$$

Here $\mu$ refers to the factorization scale between the heavy and light contributions in $\mathscr{C}_{j,(2, L)}$ and 'as' denotes the limit $Q^{2} \gg m^{2}$. The $C_{j,(2, L)}$ are precisely the light Wilson coefficients and describe all the process dependence. The arguments $\left(n_{f}\right),\left(n_{f}+1\right)$, indicate at how many light flavors the respective quantities have to be taken. This factorization is only valid if the heavy quark coefficient functions are defined in such a way that all radiative corrections containing heavy quark loops are included. Otherwise (2.2) would not show the correct asymptotic $Q^{2}$-behavior [8]. The mass dependence is given by the process independent massive OMEs $A_{i j}$, which are the flavor-decomposed twist-2 operator matrix elements

$$
A_{k i}^{\mathrm{S}, \mathrm{NS}}\left(\frac{m^{2}}{\mu^{2}}, N\right)=\left\langle i\left|O_{k}^{\mathrm{S}, \mathrm{NS}}\right| i\right\rangle_{H}=\delta_{k i}+\sum_{l=1}^{\infty} a_{s}^{l} A_{k i}^{\mathrm{S}, \mathrm{NS},(l)}\left(\frac{m^{2}}{\mu^{2}}, N\right) .
$$

Here, $i$ denotes the external on-shell particle $(i=q, g)$ and $O_{k}$ stands for the quarkonic $(k=q)$ or gluonic $(k=g)$ operator emerging in the light-cone expansion. The subscript $H$ indicates that we require the presence of heavy quarks of one type with mass $m$. The logarithmic terms in $m^{2} / \mu^{2}$ are completely determined by renormalization and contain contributions of the anomalous dimensions of the twist-2 operators. Thus at NNLO the fermionic parts of the 3-loop anomalous dimensions calculated in Refs. [9] appear. All pole terms of the unrenormalized results provide a check on our calculation and the single pole terms allow for a first independent calculation of the terms $\propto T_{F}$ of the 3-loop anomalous dimensions.

In case of the gluon operator, the contributing terms are denoted by $A_{g q, Q}$ and $A_{g g, Q}$. For the quark operator, one distinguishes whether the operator couples to a heavy or light quark. In the NS-case, the operator, by definition, couples to the light quark. Thus there is only one term, $A_{q q, Q}^{\mathrm{NS}}$. In the $\mathrm{S}$ and PS-case, two OMEs can be distinguished, $\left\{A_{q q, Q}^{\mathrm{PS}}, A_{q g, Q}^{\mathrm{S}}\right\}$ and $\left\{A_{Q q}^{\mathrm{PS}}, A_{Q g}^{\mathrm{S}}\right\}$, where, in the former case, the operator couples to a light quark and in the latter case to a heavy quark.

Eq. (2.2) allows to calculate the heavy flavor Wilson coefficients in the limit $Q^{2} \gg m^{2}$ up to $O\left(a_{s}^{3}\right)$ by combining the results obtained in Ref. [2] for the light flavor Wilson coefficients with the 3-loop massive OMEs which are computed in this work [7].

A related application of the heavy OMEs is given when using a variable flavor number scheme to describe parton densities including massive quarks. The OMEs are then the transition functions going from $n_{f}$ to $n_{f}+1$ flavors. One thus may define parton densities for massive quarks, see e.g. Ref. [8]. This is of particular interest for heavy quark induced processes at the LHC, such as $c \bar{s} \rightarrow W^{+}$at large enough scales $Q^{2}$.

\section{Renormalization}

We work in Feynman gauge and use dimensional regularization in $D=4+\varepsilon$ dimensions, applying the $\overline{\mathrm{MS}}$-scheme, if not stated otherwise. The renormalization proceeds in four steps, which we will briefly sketch here and refer to [7] for more details. Mass renormalization is performed in the on-shell scheme [10], whereas for charge renormalization we use the $\overline{\mathrm{MS}}$-scheme. We work in 
an intermediate MOM-scheme for charge renormalization by requiring that the heavy quark loop contributions to the gluon propagator vanish for on-shell external momentum. This is necessary for the renormalization of the massive OMEs to cancel infrared singularities which would otherwise remain. $Z_{g}$ in this MOM-scheme can be calculated using the background field method [11]. Finally, we transform our result back to the $\overline{\mathrm{MS}}$-scheme for coupling constant renormalization via, [7],

$$
a_{s}^{\mathrm{MOM}}=a_{s}^{\overline{\mathrm{MS}}}-\beta_{0, Q} \ln \left(\frac{m^{2}}{\mu^{2}}\right) a_{s}^{\overline{\mathrm{MS}}^{2}}+\left[\beta_{0, Q}^{2} \ln ^{2}\left(\frac{m^{2}}{\mu^{2}}\right)-\beta_{1, Q} \ln \left(\frac{m^{2}}{\mu^{2}}\right)-\beta_{1, Q}^{(1)}\right] a_{s}^{\overline{\mathrm{MS}}^{3}},
$$

with

$$
\beta_{0, Q}=-\frac{4}{3} T_{F}, \quad \beta_{1, Q}=-4\left(\frac{5}{3} C_{A}+C_{F}\right) T_{F}, \quad \beta_{1, Q}^{(1)}=-\frac{32}{9} T_{F} C_{A}+15 T_{F} C_{F} .
$$

The remaining singularities are of the ultraviolet and collinear type. The former are renormalized via the operator $Z$-factors, whereas the latter are removed via mass factorization through the transition functions $\Gamma$. After coupling- and mass renormalization, the renormalized heavy flavor OMEs are then obtained by

$$
A=Z^{-1} \hat{A} \Gamma^{-1}
$$

where quantities with a hat are unrenormalized. Note that in the singlet case operator mixing occurs and hence Eq. (3.3) should be read as a matrix equation, contrary to the NS-case. The Zand $\Gamma$-factors can be expressed in terms of the anomalous dimensions of the twist -2 operators to all orders in the strong coupling constant, cf. [7, 12] up to $O\left(a_{s}^{3}\right)$. From Eq. (3.3) one can infer that for operator renormalization and mass factorization at $O\left(a_{s}^{3}\right)$, the anomalous dimensions up to NNLO [9] together with the 1-loop massive OMEs up to $O\left(\varepsilon^{2}\right)$ and the 2-loop massive OMEs up to $O(\varepsilon)$ are needed. The 2-loop OMEs up to $O\left(\varepsilon^{0}\right)$ were calculated in Refs. [3, 5, 8, 13]. Higher orders in $\varepsilon$ enter since they multiply $Z$ - and $\Gamma$-factors containing poles in $\varepsilon$. This has been worked out in detail in Ref. [12], where we presented the $O(\varepsilon)$ terms $\bar{a}_{Q g}^{(2)}, \bar{a}_{q q, Q}^{(2), \mathrm{NS}}$ and $\bar{a}_{Q q}^{(2) \mathrm{PS}}$. The terms $\bar{a}_{g g, Q}^{(2)}$ and $\bar{a}_{g q, Q}^{(2)}$ were given in Refs. [13]. Thus all terms needed for the renormalization at 3-loops in the unpolarized case are known.

Finally we would like to point out the difference between the MOM- and $\overline{\mathrm{MS}}$-scheme for coupling constant renormalization at NLO [13]. Eq. (2.2) holds only for completely inclusive quantities, including radiative corrections containing heavy quark loops [8]. Additionally, (2.2) has to be applied in such a way that renormalization of the coupling constant is carried out in the same scheme for all quantities contributing, i.e., the $\overline{\mathrm{MS}}$-scheme. If one evaluates the heavy-quark Wilson coefficients, diagrams of the type shown in Fig. 1 may appear as well. It contains a virtual heavy quark loop correction to the gluon propagator in the initial state and contributes to the terms $L_{g, i}$ and $H_{g, i}$, respectively, depending on whether a light or heavy quark pair is produced in the final state. Note that in the former case, this diagram contributes to $F_{(2, L)}\left(x, Q^{2}\right)$ in the inclusive case, but is absent in the semi-inclusive $Q \bar{Q}$-production cross section. In Refs. [1], the coupling constant was renormalized in the MOM-scheme in $O\left(a_{s}^{2}\right)$ by absorbing the contributions of the above diagram into the coupling constant. This can be made explicit by considering the complete gluonic Wilson coefficient up to $O\left(a_{s}^{2}\right)$, including one heavy quark, see Eq. (2.2),

$$
C_{g, 2}\left(n_{f}\right)+L_{g, 2}\left(n_{f}+1\right)+H_{g, 2}\left(n_{f}+1\right)=a_{s}^{\overline{\mathrm{MS}}}\left[A_{Q g}^{(1), \overline{\mathrm{MS}}}+C_{g, 2}^{(1)}\left(n_{f}+1\right)\right]
$$




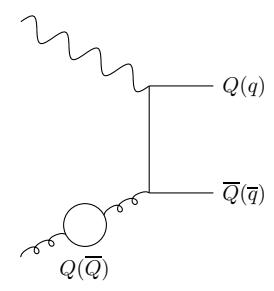

Figure 1: $O\left(a_{s}^{2}\right)$ virtual heavy quark corrections to $\mathrm{H}_{g,(2, L)}^{(2)}$.

$$
+a_{s}^{\overline{\mathrm{MS}}^{2}}\left[A_{Q g}^{(2), \overline{\mathrm{MS}}}+A_{Q g}^{(1), \overline{\mathrm{MS}}} C_{q, 2}^{(1), \mathrm{NS}}\left(n_{f}+1\right)+A_{g g, Q}^{(1), \overline{\mathrm{MS}}} C_{g, 2}^{(1)}\left(n_{f}+1\right)+C_{g, 2}^{(2)}\left(n_{f}+1\right)\right] .
$$

The above equation is given in the $\overline{\mathrm{MS}}$-scheme. Here, the diagram shown in Fig. 1 contributes, corresponding exactly to the color factor $T_{F}^{2}$. Transformation to the MOM-scheme for $a_{s}$, Eq. (3.1), yields

$$
\begin{aligned}
& C_{g, 2}\left(n_{f}\right)+L_{g, 2}\left(n_{f}+1\right)+H_{g, 2}\left(n_{f}+1\right)=a_{s}^{\text {мом }}\left[A_{Q g}^{(1), \text { мом }}+C_{g, 2}^{(1)}\left(n_{f}+1\right)\right] \\
& +a_{s}^{\text {мом } 2}\left[A_{Q g}^{(2), \text { мом }}+A_{Q g}^{(1), \text { MOм }} C_{q, 2}^{(1), \mathrm{NS}}\left(n_{f}+1\right)+C_{g, 2}^{(2)}\left(n_{f}+1\right)\right] .
\end{aligned}
$$

In the above equation, all contributions due to diagram 1 have canceled, i.e. the color factor $T_{F}^{2}$ does not occur at the 2-loop level in the MOM-scheme. Splitting up Eq. (3.5) into $H_{g, i}$ and $L_{g, i}$, one observes that $L_{g, i}$ vanishes at $O\left(a_{s}^{2}\right)$. The term $H_{g, i}$ is the one calculated in Ref. [3], which is the asymptotic expression of the gluonic heavy flavor Wilson coefficient as calculated exactly in Refs. [1]. It is not clear whether the same can be achieved at the 3-loop level as well, i.e., transforming the general inclusive factorization formula (2.2) in such a way that only the contributions due to heavy flavors in the final state remain. Therefore one should use the asymptotic expressions at 3 loops only for completely inclusive analyzes. This approach has also been adopted in Ref. [8] for the renormalization of the massive OMEs, which was performed in the $\overline{\mathrm{MS}}$-scheme and not in the MOM-scheme, as previously in Ref. [3]. In the NS-case a similar argument holds, which can be found in Ref. [3].

\section{Calculation and Results}

The massive OMEs at $O\left(a_{s}^{3}\right)$ are given by 3-loop self-energy type diagrams, which contain a local operator insertion. The external massless particles are on-shell. The heavy quark mass sets the scale and the spin of the local operator is given by the Mellin-variable $N$. The steps for the calculation are the following: We use QGRAF [14] for the generation of diagrams. Approximately 2700 diagrams contribute to all the OMEs. For the calculation of the color factors we refer to [15]. The diagrams are then genuinely given as tensor integrals. Applying a suitable projector provides the results for the specific Mellin moment under consideration. The diagrams are further translated into a form, which is suitable for the program MATAD [16], through which the expansion in $\varepsilon$ is performed and the corresponding massive three-loop tadpole-type diagrams are calculated. We have implemented all these steps into a FORM-program [17] and checked our procedures against various complete two-loop results and certain scalar 3-loop integrals and found full agreement. 
Applying Eq. (3.3), one can predict the pole structure of the unrenormalized results and thus the logarithmic terms of the renormalized OMEs. These contributions can be expressed in terms of the anomalous dimensions up to 3 loops, the expansion coefficients of the QCD $\beta$-function up to 2 loops and the 1- and 2-loop contributions to the massive OMEs. Thus the logarithmic terms are known for general values of $N$. This is not the case for the constant term, which contains the genuine 3-loop contributions $a_{i j}^{(3)}$. These are known for the fixed values of $N$ as calculated in this work [7].

For the OMEs $A_{Q g}^{(3)}, A_{q g, Q}^{(3)}$ and $A_{g g, Q}^{(3)}$ the moments $N=2$ to 10 , for $A_{Q q}^{(3), \mathrm{PS}}$ to $N=12$, and for $A_{q q, Q}^{(3), \mathrm{NS}}, A_{q q, Q}^{(3), \mathrm{PS}}, A_{g q, Q}^{(3)}$ to $N=14$ were computed. For the flavor non-singlet terms, we calculated as well the odd moments $N=1$ to 13 , corresponding to the light flavor--combinations. ${ }^{1}$ The complete calculation took about 250 days of computer time. All our results agree with the predictions obtained from renormalization, providing us with a strong check on our calculation. As an example, we show the constant term of $a_{Q g}^{(3)}$ of the unrenormalized OME $A_{Q g}^{(3)}$ for $N=10$

$$
\begin{aligned}
a_{Q g}^{(3)}(10)= & T_{F} C_{A}^{2}\left(\frac{6830363463566924692253659}{685850575063965696000000}-\frac{563692}{81675} \mathrm{~B}_{4}+\frac{483988}{9075} \zeta_{4}-\frac{103652031822049723}{415451499724800} \zeta_{3}-\frac{20114890664357}{581101290000} \zeta_{2}\right) \\
& +T_{F} C_{F} C_{A}\left(\frac{872201479486471797889957487}{2992802509370032128000000}+\frac{1286792}{81675} \mathrm{~B}_{4}-\frac{643396}{9075} \zeta_{4}-\frac{761897167477437907}{33236119977984000} \zeta_{3}+\frac{15455008277}{660342375} \zeta_{2}\right) \\
& +T_{F} C_{F}^{2}\left(-\frac{247930147349635960148869654541}{148143724213816590336000000}-\frac{11808}{3025} \mathrm{~B}_{4}+\frac{53136}{3025} \zeta_{4}+\frac{9636017147214304991}{7122025709568000} \zeta_{3}+\frac{14699237127551}{15689734830000} \zeta_{2}\right) \\
& +T_{F}^{2} C_{A}\left(\frac{23231189758106199645229}{633397356480430080000}+\frac{123553074914173}{5755172290560} \zeta_{3}+\frac{4206955789}{377338500} \zeta_{2}\right) \\
& +T_{F}^{2} C_{F}\left(-\frac{18319931182630444611912149}{1410892611560158003200000}-\frac{502987059528463}{113048027136000} \zeta_{3}+\frac{24683221051}{46695639375} \zeta_{2}\right)-\frac{896}{1485} T_{F}^{3} \zeta_{3}+n_{f} T_{F}^{2} C_{A}\left(\frac{297277185134077151}{15532837481700000}\right. \\
& \left.-\frac{1505896}{245025} \zeta_{3}+\frac{189965849}{188669250} \zeta_{2}\right)+n_{f} T_{F}^{2} C_{F}\left(-\frac{1178560772273339822317}{107642563748181000000}+\frac{62292104}{13476375} \zeta_{3}-\frac{49652772817}{93391278750} \zeta_{2}\right) .
\end{aligned}
$$

Here $\zeta_{i}$ denotes the Riemann $\zeta$-function at integer argument $i$ and the term $B_{4}$ is given by

$$
\mathrm{B}_{4}=-4 \zeta_{2} \ln ^{2} 2+\frac{2}{3} \ln ^{4} 2-\frac{13}{2} \zeta_{4}+16 \mathrm{Li}_{4}\left(\frac{1}{2}\right)
$$

It appears in all OMEs we calculated and is known to arise as a genuine mass effect.

\section{Conclusions and Outlook}

We calculated all massive 3-loop OMEs for even Mellin-moments $N=2 \ldots 10(12,14)$ using MATAD. This confirms for the first time, in an independent calculation, the moments of the fermionic parts of the corresponding 3-loop anomalous dimensions [9]. Combining our results with [2], this provides fixed moments of the heavy flavor Wilson coefficients of $F_{2}$ in the limit $Q^{2} \gg m^{2}$. First phenomenological studies of the effects of our calculation are in preparation and will be used extending the heavy flavor treatment from 2- to 3-loop accuracy in foregoing analyzes [20].

\footnotetext{
${ }^{1}$ The massive OMEs for transversity have been calculated in [18]. The corresponding anomalous dimensions found in [19] are confirmed in the color factors appearing in the present calculation and extended to higher values of $N$.
} 


\section{Acknowledgments}

We would like to thank K. Chetyrkin, J. Smith, M. Steinhauser and J. Vermaseren for useful discussions. We thank both IT groups of DESY providing us access to special facilities to perform the present calculation.

\section{References}

[1] E. Laenen, et al., Nucl. Phys. B 392 (1993) 162; S. Riemersma, J. Smith and W. L. van Neerven, Phys. Lett. B 347 (1995) 143 [hep-ph/9411431].

[2] J. A. M. Vermaseren, A. Vogt and S. Moch, Nucl. Phys. B 724 (2005) 3 [hep-ph/0504242].

[3] M. Buza, et al., Nucl. Phys. B 472 (1996) 611 [hep-ph/9601302].

[4] J. Blümlein and W. L. van Neerven, Phys. Lett. B 450 (1999) 417 [arXiv:hep-ph/9811351].

[5] I. Bierenbaum, J. Blümlein and S. Klein, Nucl. Phys. B 780 (2007) 40 [hep-ph/0703285]; Phys. Lett. B 648 (2007) 195 [hep-ph/0702265].

[6] J. Blümlein, et al. Nucl. Phys. B 755 (2006) 272 [hep-ph/0608024].

[7] I. Bierenbaum, J. Blümlein and S. Klein, Nucl. Phys. B 820 (2009) 417 [hep-ph/0904.3563].

[8] M. Buza, et al., Eur. Phys. J. C 1 (1998) 301 [hep-ph/9612398].

[9] S. A. Larin, T. van Ritbergen and J. A. M. Vermaseren, Nucl. Phys. B 427 (1994) 41; S. A. Larin, et al., Nucl. Phys. B 492 (1997) 338 [hep-ph/9605317]; A. Retey and J. A. M. Vermaseren, Nucl. Phys. B 604 (2001) 281 [hep-ph/0007294]; J. Blümlein and J. A. M. Vermaseren, Phys. Lett. B 606 (2005) 130 [hep-ph/0411111]; S. Moch, J. A. M. Vermaseren and A. Vogt, Nucl. Phys. B 688 (2004) 101 [hep-ph/0403192]; Nucl. Phys. B 691 (2004) 129 [hep-ph/0404111].

[10] D. J. Broadhurst, et al. Z. Phys. C 48 (1990) 673; 52 (1991) 111.

[11] L. F. Abbott, Nucl. Phys. B 185 (1981) 189.

[12] I. Bierenbaum, et al., Nucl. Phys. B 803 (2008) 1 [hep-ph/0803.0273];

[13] I. Bierenbaum, J. Blümlein and S. Klein, Phys. Lett. B 672 (2009) 401 [hep-ph/0901.0669].

[14] P. Nogueira, J. Comput. Phys. 105 (1993) 279.

[15] T. van Ritbergen, A. N. Schellekens and J. A. M. Vermaseren, Int. J. Mod. Phys. A 14 (1999) 41 [hep-ph/9802376].

[16] M. Steinhauser, Comput. Phys. Commun. 134 (2001) 335 [hep-ph/0009029] and MATAD 3.0.

[17] J. A. M. Vermaseren, New features of FORM, [math-ph/0010025].

[18] J. Blümlein, S. Klein and B. Tödtli, arXiv:0909.1547 [hep-ph], Phys. Rev. D in print; arXiv:0909.1487 [hep-ph].

[19] J. A. Gracey, Nucl. Phys. B 662 (2003) 247 [arXiv:hep-ph/0304113]; Nucl. Phys. B 667 (2003) 242 [arXiv:hep-ph/0306163]; JHEP 0610 (2006) 040 [arXiv:hep-ph/0609231]; Phys. Lett. B 643 (2006) 374 [arXiv:hep-ph/0611071].

[20] J. Blümlein, H. Böttcher and A. Guffanti, Nucl. Phys. B 774 (2007) 182 [arXiv:hep-ph/0607200]; Nucl. Phys. Proc. Suppl. 135 (2004) 152 [arXiv:hep-ph/0407089];

S. Alekhin, J. Blümlein, S. Klein and S. Moch, arXiv:0908.2766 [hep-ph], Phys. Ref. D in print. 